\title{
Application of Chest CT in the Primary Screening of COVID-19 Related High- Risk Population
}

\author{
Ting-Ting Zhou ${ }^{1},{ }^{2}{ }^{*}$, Feng-Xian Wei ${ }^{1}$ and Zhao Liu ${ }^{3}$ \\ 1Lanzhou University Second Clinical Medical College, Lanzhou University, Lanzhou, 730030 Gansu, China \\ ${ }^{2}$ Department of Respiratory Medicine, Lanzhou University Second Hospital, Lanzhou, 730030 Gansu, China \\ ${ }^{3}$ Department of Radiology, The first affiliation Hospital of Lanzhou University, Lanzhou, 730030 Gansu, China
}

${ }^{*}$ Corresponding author: Ting-ting Zhou, Lanzhou University Second Clinical Medical College, Lanzhou University Cuiyingmen 82, Chengguan District, Lanzhou, 730030 Gansu, China, Tel: ++86-0931-5190524; E-mail: zhoutt20@126.com

Received date: April 18, 2020; Accepted date: June 05, 2020; Published date: June 13, 2020

Citation: Zhou TT, Wei FX, Liu Z (2020) Application of Chest CT in the Primary Screening of COVID-19 Related High-Risk Population. Arch Clin Microbiol Vol. 11 No. 3:112

Copyright: (C) 2020 Zhou TT, et al. This is an open-access article distributed under the terms of the Creative Commons Attribution License, which permits unrestricted use, distribution, and reproduction in any medium, provided the original author and source are credited.

\section{Abstract}

Although nucleic acid test and immunity antibody test are the only standards for the diagnosis of Coronavirus Disease 2019 (COVID-19), while it was rarely applied for primary screening in all COVID-19 related close contacts. The management of such kind of population still need additional method besides medical observation. Chest CT as a routinely examination in most hospital, it has certain advantages and limitation in the management of COVID-19. Here, we summarized and commented the role of chest by clinical perspective in the primary screening.

\section{Keywords: coVID-19, Chest CT, Primary Screening}

\section{Abbreviations:}

COVID-19: Coronavirus Disease 2019; CT: Computed tomography

\section{Introduction}

Coronavirus Disease 2019 (COVID-19) was first reported in China on 31 December 2020 and was declared a Public Health Emergency of International Concern on 30 January 2020. While now, it has been spread worldwide with nearly two million cases were reported to be infected, and the overall estimated fatality rate was about $7 \%$. In clinical practice, chest CT is most of the most important as well as the commonest examination recommended by the doctors. Although its diagnostic role in COVID-19 was weaken in current clinical management in most areas because of the sufficient and quick nucleic acid tests. Chest CT still plays certain roles in the management of all COVID-19 related high-risk population without clear implication of nucleic acid because of its specific characteristics as below [1].

\section{Specific Advantages of Chest CT}

Chest CT as one of the most important references for the infections presented as viral pneumonia, there is no doubt that it plays vital roles in the management of COVID-19. Chest CT have rapid process, high sensitivity, low exposure risk and repeatability in the screening of COVID-19. As a conventional and non-invasive imaging modality, more than 300 cases can be checked by one CT machine every day in routinely equipped hospital. Its process is simple and can be guided by broadcast in developing areas or a guidance robot in developed areas, thus the exposure risk of doctors can be significantly reduced compared with nucleic acid test. The images can be stored and re-read by physicians and multidiscipline experts and can be used for dynamic monitoring for both diagnosis and treatment effects. Meanwhile, the sensitivity of chest CT imaging was significantly higher than initial nucleic acid tests from swab samples in one time [2]. In all editions of the Diagnosis and Treatment Guidelines for COVID-19 by The National Health Commission of China [3], chest CT imaging is always one of the three clinical manifestations for the diagnosis of suspected case. Therefore, chest CT has specific advantages in the primary screening of COVID-19 related high risk population by clinical perspective.

\section{Varied Accuracy of Chest CT}

However, additional suggestions should be attentional for its clinical application. Obviously varied diagnostic accuracy of chest CT were reported from different centers. After systematic reviewing relevant literature on chest CT with nucleic acid test as a reference in the differential diagnosis of COVID-19 from other pneumonia patients, 2 studies containing 1396 cases were included. The sensitivity and specificity were reported to be $95 \%$ and $70 \%$ in a cohort containing 382 cases [4], while $97 \%$ and $25 \%$ in the other cohort containing 1014 cases [2], respectively. Another study analyzed the performance of experts, including 3 Chinese radiologists and 4 
U.S. radiologists, had sensitivities of $80 \%, 67 \%, 97 \%, 93 \%, 83 \%$, $73 \%$ and $70 \%$ and specificities of $100 \%, 93 \%, 7 \%, 100 \%, 93 \%$, $93 \%, 100 \%$ after blindly reviewing chest CTs [5]. Based on this fact, radiologists maybe need to be trained for such new disease, and discussion or expert consultation may be necessary for highly suspected case.

\section{Clinical Suggestions of Chest CT}

Comprehensive assessment of epidemiological exposure, related clinical symptoms and routine laboratory tests were advised to be completed despite of negative CT findings $[1,6,7]$. Especially, the assessment of epidemiological exposure served as the most important part and first step in the diagnosis of COVID-19, which was an independently high-risk factor and diagnostic reference of "suspected case". The diagnostic value of chest CT would be improved only when it was combined analyzed with other clinical manifestations. For population in high-risk areas with unclear epidemiological history or low-to-medium risk of epidemiological exposure, chest CT scan deserve as a screening tool with comprehensive assessment. whereas, it should be noticed that once a case was found to be with high risk of epidemiological exposure, and nucleic acid test should be recommended first other than chest $\mathrm{CT}[8]$.

\section{Potential Application of Chest CT for Asymptomatic Infections}

At last, more and more concerns as well as attention focused on asymptomatic infections currently. Such kinds of cases were mostly because of epidemiological history of close contacts with previously COVID-19 infections. In the seventh edition of Guidelines in China, the epidemiological history was defined as: (1) a history of travel or residence in Wuhan city and surrounding areas, or other communities where COVID-19 cases had been reported in the last 14 days before symptom onset; (2) a history of contact with SARS-CoV-2 infectious cases (with positive nucleic acid test); (3) a history of contacting with patients with fever or respiratory symptoms from Wuhan city and surrounding areas, or other communities where COVID-19 had been reported in the last 14 days before symptom onset; (4) a history of contacting with cluster of confirmed cases (2 cases with fever and/or respiratory symptoms occurred within 2 weeks in small areas, such as home, office, class of school, etc.) [3]. A study including 24 asymptomatic infections showed positive chest CT findings in early stage in 17 cases $(70.8 \%)$ before positive nucleic acid results [9]. This indicated a possibility of applying chest CT for monitoring in all close contacts with low-to-medium risk of epidemiological exposure besides of voluntary isolation. Often nucleic acid test would not be applying for "close contact" judged as low-to-medium exposure risk, however we concerned that only isolation with observing related clinical symptoms may be not enough in status.
In conclusion, Chest CT has specific advantages with high sensitivity in clinic practice. While, comprehensive assessment especially focusing on epidemiological history was required in the primary screening of COVID-19 related high-risk population.

\section{Declarations}

\section{Acknowledgement}

The opinions expressed here are solely those of the author, and not of his affiliations.

\section{Funding}

None.

\section{Conflict of interest}

The author has no conflict of interest related to this publication.

\section{References}

1. Li B, Li X, Wang Y (2019) Diagnostic Value and Key Features of Computed Tomography in Coronavirus Disease 2019. Emerge Microbes Infect 1-14.

2. Ai T, Yang Z, Hou H (2020) Correlation of Chest CT and RT-PCR Testing in Coronavirus Disease 2019 (COVID-19) in China: A Report of 1014 Cases Radiology 200642.

3. General Office of National Health Committee, Office of State Administration of Traditional Chinese Medicine (2020) Notice on the issuance of a program for the diagnosis and treatment of COVID-19.

4. Xiong Z, Fu L, Zhou H (2020) Construction and Evaluation of a Novel Diagnosis Process for 2019-Corona Virus Disease.

5. Bai HX, Hsieh B, Xiong Z (2020) Performance of radiologists in differentiating COVID-19 from viral pneumonia on chest CT [published online ahead of print, 2020 Mar 10]. Radiology.

6. Zhou TT, Wei FX (2020) Primary stratification and identification of suspected Corona virus disease 2019 (COVID-19) from clinical perspective by a simple scoring proposal. Mil Med Res 7: 16.

7. Li K, Fang Y, Li W (2020) CT image visual quantitative evaluation and clinical classification of coronavirus disease (COVID-19).

8. Scott SE, Zabel K, Collins J (2020) First Mildly III, NonHospitalized Case of Coronavirus Disease 2019 (COVID-19) Without Viral Transmission in the United States - Maricopa County, Arizona.

9. $\mathrm{Hu} \mathrm{Z}$, Song C, Xu C (2020) Clinical characteristics of 24 asymptomatic infections with COVID-19 screened among close contacts in Nanjing, China. Sci China Life Sci. 